\title{
Efficacy of hypnotherapy on pain intensity and psychological distress among women with premenstrual dysphoric disorder, applicability of suggestions focused on cognitive flexibility and ego strength
}

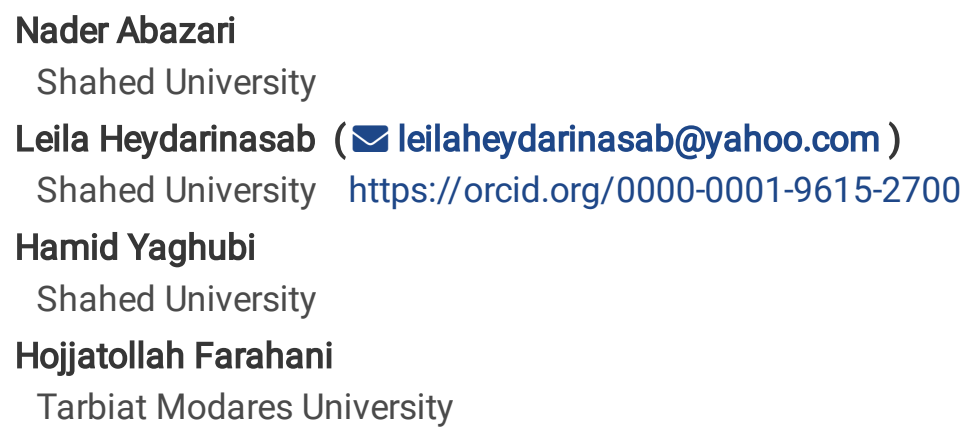




\section{Abstract}

Background: Premenstrual dysphoric disorder PMDD is a health difficulty that is so similar to premenstrual syndrome PMS but is more serious Many women with PMDD may also have anxiety or depression, in this way, lasting irritability or anger that may affect other people, feelings of sadness or despair, or even thoughts of suicide, feelings of tension or anxiety, panic attacks, mood swings or crying often, lack of interest in daily activities and relationships, trouble thinking or focusing, tiredness or low energy, food cravings or binge eating, trouble sleeping, feeling out of control, physical symptoms such as cramps, bloating, breast tenderness, headaches, and joint or muscle pain are some of prevalent symptoms suffer women with PMDD

Methods: The present research was a randomized controlled trial. In order to control variables pain intensity and psychological distress among patients with PMDD, the participants were selected accidently from female students of Shahed University of Tehran who consulted to psychology in order to assess their premenstrual symptoms and randomly divided into two groups. Each of groups encompassed 30 women between 20 up to 35-year-old whom diagnosed by PMDD via PSST from winter 2019 to spring 2020. In this study, one group received hypnotherapy and the other group received no intervention.

Results: There are significant differences between the two groups. The efficacy of the procedure of hypnotherapy in the experimental group was more than that in the control group, $(\mathrm{sig}=0.05)$.

Conclusion The present study findings revealed that hypnotherapy with suggestions focused on cognitive flexibility and ego strength affected pain intensity and psychological distress among females with PMDD significantly. (sig=0.05)

\section{Background}

Premenstrual dysphoric disorder PMDD is a health difficulty that is so similar to premenstrual syndrome PMS but is more serious(1). PMDD causes severe irritability, depression, or anxiety in the week or two days before menstruation. Symptoms normally go away two to three days after menstruation starts(2). PMDD affects up to $8 \%$ of women of childbearing age(3). Many women with PMDD may also have anxiety or depression, in this way, lasting irritability or anger that may affect other people, feelings of sadness or despair, or even thoughts of suicide, feelings of tension or anxiety, panic attacks, mood swings or crying often, lack of interest in daily activities and relationships, trouble thinking or focusing, tiredness or low energy, food cravings or binge eating, trouble sleeping, feeling out of control, physical symptoms such as cramps, bloating, breast tenderness, headaches, and joint or muscle pain are some of prevalent symptoms suffer women with PMDD(4). Researchers do not know for sure what causes PMDD or PMS. Hormonal changes throughout the menstrual cycle may play a role, a brain chemical called serotonin may also play a role in $\operatorname{PMDD}(5)$.

Previous studies showed that biological, psychological, and social-cultural factors cause PMDD(4). These factors include educational and marital status, genetic predisposition, age, pain during menses, amount of menstrual bleeding, history of physical and psychiatric illness, treatment seeking behavior, history of traumatic event, sleeping hour, physical exercise, and maternal history of PMDD(6). PMDD has been associated with suicide and accident rate, major depressive disorder, high job absenteeism, poor quality of life, and poor family and coworkers relationship(5). One of the most effective factors can predict intensity of symptoms of PMDD is flexibility in thought changing and selecting different attitudes toward objects(7). it has been shown that psychological maturity, coping styles and self-perception can significantly affect incidence and intensity of PMDD symptoms(1).

Researches have demonstrated that pain is most common complaint among menstruating women; it has some different difficulties for them(8). For instance, generally, PMDD increases perceived pain among women; it dramatically augments the risk of pain medication abuse(3). In this way, it has been reported that psychological distress is another most important symptom women suffer from before and during menstruation(1). After the complaints about pain, psychological distress is 
the most common symptom that makes women consult with a mental health practitioner(2). It has been observed that pain intensity can be affected by psychosocial variables such as pain catastrophizing, beliefs on pain, coping skills, perceived social support, and learning about pain from important others(5).

Women with PMDD have decreased emotional adjusting more in the premenstrual phase than controls had done (3, 8).among these women, emotional adjusting is negatively associated with depression, anxiety, and stress(5). The premenstrual estrogen negatively correlated with anxiety and stress in women with PMDD(1). So do, there is significant association between PMDD experience and academic performance, moreover, the length of menses significantly associated with academic performance(2).

Several etiological causes have been introduced to explain the disorders, which in turn have led to different treatment methods to control the clinical symptoms(9). hypnosis treatment can attenuate the stress caused by the syndrome and remove the environmental background of the disorder to a large extent(10). hypnosis is a condition that results from attention, acceptance, and concentration, in which there must be degrees of three facets of the same time: decomposition or abstraction, absorption, inclusiveness(11).hypnotherapy is based on the assumption that most psychological disturbances are a negative form of self-hypnosis, so that negative thoughts are accepted critically and even without informed knowledge(12). hypnotherapy involves several basic methods including relaxation, guided imagery, cognitive reconstruction, gradual desensitization, and the training of hypnosis skills(13). With regard to suggested theories on formation of PMDD, it looks to be effective to use hypnotherapy to interevent effective psychological factors of PMDD(9).

In this investigation we aimed to analyze effectiveness of hypnotherapy on pain intensity and psychological distress with regard to applicability of suggestions focused on cognitive flexibility and ego strength.

\section{Method}

The present research was a randomized controlled trial. In order to control variables pain intensity and psychological distress among patients with PMDD, the participants were selected accidently from female students of Shahed University of Tehran who consulted to psychology in order to assess their premenstrual symptoms and randomly divided into two groups. Each of groups encompassed 30 women between 20 up to 35-year-old whom diagnosed by PMDD via PSST from winter 2019 to spring 2020. In this study, one group received hypnotherapy and the other group received no intervention. All of 60 participants conformed to the research criteria and none of them were excluded from analysis process. In order to control the obtrusive variables, we matched the groups in terms of demographic variables. The inclusion criteria were age ranging between 20 and 35-year-old and PMDD diagnosis. The exclusion criteria were psychotic disorder diagnosis, menopause and lack of cooperation in the therapeutic process. The present study was reviewed and approved by the institutional review board of Shahed University of Tehran. In order to analyze data, repeated measures analysis of variance (ANOVA) was applied in SPSS software (version 19, SPSS Inc., Chicago, IL, USA). In addition, repeated measurements were taken during the therapy. To determine the baseline, a pretest was conducted on the first session, mid-test and posttest, also a follow up test 2 months later. The level of significance in this investigation was 0.05 . The present study was reviewed and approved by the Institutional Review Board (IRB) of Shahed University under the code of IR.SHAHED.REC.1399.118 and was registered by the Iranian Randomized Controlled Trials Site under the code of IRCT20201110049332N1

\section{Pain intensity}

The Numerical Rating Scale (NRS) was used in the present study to measure pain intensity. The NRS was made by McCaffery in 1999. The NRS is a unidimensional instrument of pain intensity and includes its total score ranges between 1 and 10, where 1 means no pain and 10 mean the most severe pain. This instrument has been supported by the National Institute of Pain in the USA for the measurement of pain intensity. This instrument is user-friendly and the patients do not 
require powerful visible ability or high literacy to use it. According to literature, this tool has suitable validity and reliability. Different investigations have estimated its reliability to be about $0.84,0.89$, and $0.92 .12 \mathrm{NRS}$ scores strongly correlated to visual analogue scale (VAS) scores at all time periods $(r=0.94,95 \% \mathrm{Cl}=0.93$ to 0.95$)$. The slope of the regression line was $1.01(95 \% \mathrm{Cl}=0.97$ to 1.06$)$ and the $y$-intercept was $-0.34(95 \% \mathrm{Cl}=-0.67$ to -0.01$)$. The minimum clinically significant difference in pain was $1.3(95 \% \mathrm{Cl}=1.0$ to 1.5$)$ and $1.4(95 \% \mathrm{Cl}=1.1$ to 1.7$)$ on the NRS and VAS, respectively(14).

\section{Psychological distress (DASS-42)}

The Depression Anxiety and Stress Scales (DASS-42) has 3 elements, including depression, anxiety, and stress, and contains 42 questions. This tool was made by Lovibond in 1995 in order to measure the common signs of stress, anxiety, and depression. This tool measures the mental situation in the previous two weeks. Each element contains 14 questions, and the degree of each element is determined by computing the sum of the scores of these questions. The reliability of this instrument in Iran has been estimated about 0.95, 0.92, and 0.97 .14 In another investigation, it has been reported that the DASS-42 had very good Cronbach's alpha with amounts of $0.84,0.74$, and 0.79 for depression, anxiety, and stress, respectively. In addition, it had good factor loading values for most items ( 0.39 to 0.73 ). 15 Correlations among scales were between 0.54 and 0.68 . This tool can measure psychological distress in the continuum of normal, mild, severe, and extremely severe(15).

\section{premenstrual symptoms screening tool (PSST)}

The PSST is a quick and clinician-friendly self-report screening instrument for PMS/PMDD which also rates the impact of premenstrual symptoms on daily activities. This tool can identify women who meet full DSM-IV criteria for PMDD as well as women who experience PMS but do not qualify for a diagnosis of PMDD. Therefore, the PSST identifies women who would benefit from a more in-depth diagnostic interview for the diagnosis of PMDD, without the need to monitor symptoms over two reproductive cycles. The PSST had adequate internal consistency (Cronbach's alpha $=0.91$ ) and test-retest reliability. The PSST also had adequate convergent/discriminant validity, without redundancy(16).

\section{The Stanford Hypnotic Susceptibility Scale}

The Stanford Hypnotic Susceptibility Scale were devised in the late 1950s by Stanford University psychologists André M. Weitzenhoffer and Ernest R. Hilgard and are still used today to determine the extent to which a subject respond to hypnosis. Stanford scales scoring ranges from 0 , for individuals who do not respond to any of the hypnotic suggestions, to 12 , for those who pass all of them. Most people score in the middle range (between 5 and 7); 95 percent of the population receives a score of at least 1. Cronbach's alpha coefficient was used to measure the internal reliability of the scale. Cronbach's alpha coefficient for the total scale was equal to 0.79 , and it was $0.45,0.44$, and 0.66 for the subscales of perceptive-cognitive abilities, sensory-motor phenomena, and cognitive distortions; respectively. These coefficients for the total scale were 0.75 , and they were equal to $0.51,0.52$, and 0.35 for the subscales of perceptive-cognitive abilities, sensory-motor phenomena, and cognitive distortions, respectively(17).

\section{The process of therapy:}

In this process, we selected the patients accidentally with respect to the inclusion criteria. The participants underwent 8 sessions of hypnotherapy. Each hypnotherapy session lasted about 60 minutes. The protocol of hypnotherapy contained 8 sessions of hypnotherapy. This protocol was in accordance with the manual outlined by Hammond (1990). The hypnotherapy protocol was implemented individually. The first session is allocated to introduction and pretest in order to determine the baselines. Session 1 included familiarization, socialization, and preparation of the patient for a deep and flowing trance. Then, the trance is induced through progressive muscle relaxation (PMR); the patient enters a trance state and eventually returns to a normal state. In session 2, induction of the main suggestions is suggested including restructuring attitude and cognitive flexibility, and improving ego strength. Then, participants gradually return to the normal state. In session 3, after induction of a trance and suggesting a change in the sense of pain, suggestions are made on tolerating bad sensations (all negative sensations not only pain), Finally, the patient returns to a normal state. In session 4- 
6, after changing the sense pain and increasing the tolerance of pain, suggestions on ego strength are presented for the patient to promote beliefs on living normally like others. Session 7 included the induction of all of the previous suggestions, fixation of conditioning, prepare the patient for termination of therapeutic sessions in the next session, and gradual return to normal state. Fixation of conditioning is also the main task of session 8; in addition, the patients receive feedbacks on their sensations. Moreover session 5 and 8 was allocated to take mid-test and posttest. After the end of the trance, recommendations are presented to fix the post-hypnotic suggestions. The protocol of hypnotherapy was implemented by PhD students of clinical psychology who were educated in hypnotherapy in the Iranian Association of Hypnotism.

\section{Results}

The sample consisted of 60 patients who were randomly divided into two groups. Their age range was 20 to 35 years and their mean (standard deviation) age was 25.36 (2.7). The mean (standard deviation) of suggestibility was 9.9 (1.16). The majority of participants were single; only 9 of them were married and 51 of them were single. Moreover, 20 (33.3\%) patients of them were B.A student, 31 (51.7) of them were M.A student and finally 9 (15\%) of them were PhD student. it should be mentioned that $3(5 \%)$ of them had off springs and 57 (95\%) of them had not. The result of Mauchly's test of sphericity for pain intensity was $0.959(P=0.797)$, for DASS-42 (Depression) was $0.459(P=0)$, for DASS-42 (Anxiety) was $0.890(P=$ 0.250), for DASS-42 (Stress) was $0.714(P=0.002)$. in the following table (Table 1 ) has reported mean and standard deviation of DASS subscale Depression, DASS subscale Anxiety and DASS subscale Stress during pretest, posttest and follow up test. After that in the next table (Table 2) has been shown mean and standard deviation of pain intensity. 
Table 1

Mean and standard deviation of DASS (N: Each group $=30$ )

\begin{tabular}{|c|c|c|c|}
\hline Group & & Mean & St. Deviation \\
\hline \multirow[t]{3}{*}{ Pretest Depression } & Hypnotherapy & 33.66 & 2.50 \\
\hline & Control & 31.26 & 1.87 \\
\hline & Total & 32.46 & 2.50 \\
\hline \multirow[t]{3}{*}{ Mid-test Depression } & Hypnotherapy & 23.53 & 6.29 \\
\hline & Control & 32.53 & 1.88 \\
\hline & Total & 28.03 & 6.46 \\
\hline \multirow[t]{3}{*}{ Posttest Depression } & Hypnotherapy & 3.90 & 1.91 \\
\hline & Control & 32.06 & 1.98 \\
\hline & Total & 17.98 & 14.33 \\
\hline \multirow[t]{3}{*}{ Follow up test Depression } & Hypnotherapy & 4.86 & 1.77 \\
\hline & Control & 31.43 & 1.88 \\
\hline & Total & 18.15 & 13.51 \\
\hline \multirow[t]{3}{*}{ Pretest Anxiety } & Hypnotherapy & 32.80 & 2.38 \\
\hline & Control & 30.96 & 1.69 \\
\hline & Total & 31.88 & 2.24 \\
\hline \multirow[t]{3}{*}{ Mid test Anxiety } & Hypnotherapy & 23.30 & 2.01 \\
\hline & Control & 33.10 & 1.70 \\
\hline & Total & 28.20 & 5.27 \\
\hline \multirow[t]{3}{*}{ Posttest Anxiety } & Hypnotherapy & 4.20 & 2.23 \\
\hline & Control & 32.40 & 1.73 \\
\hline & Total & 18.30 & 14.35 \\
\hline \multirow[t]{3}{*}{ Follow up test Anxiety } & Hypnotherapy & 5.73 & 2.55 \\
\hline & Control & 31.76 & 1.94 \\
\hline & Total & 18.75 & 13.31 \\
\hline \multirow[t]{3}{*}{ Pretest Stress } & Hypnotherapy & 32.06 & 1.96 \\
\hline & Control & 30.90 & 1.34 \\
\hline & Total & 31.48 & 1.77 \\
\hline \multirow[t]{3}{*}{ Mid-test Stress } & Hypnotherapy & 22.63 & 2.18 \\
\hline & Control & 32.53 & 1.92 \\
\hline & Total & 27.58 & 5.39 \\
\hline \multirow[t]{2}{*}{ Posttest Stress } & Hypnotherapy & 4.36 & 1.92 \\
\hline & Control & 32.93 & 1.46 \\
\hline
\end{tabular}

Page $6 / 13$ 


\begin{tabular}{|llll|}
\hline Group & & Mean & St. Deviation \\
\hline \multirow{2}{*}{ Follow up test Stress } & Total & 18.65 & 14.50 \\
\hline & Hypnotherapy & 5.03 & 3.75 \\
\hline & Control & 31.23 & 1.63 \\
\hline & Total & 18.13 & 13.51 \\
\hline
\end{tabular}

Table 2

Mean and standard deviation of Pain Intensity ( $\mathrm{N}$ : Each group = 30)

\begin{tabular}{|llll|}
\hline Group & & Mean & St. Deviation \\
\hline Pretest Pain & Hypnotherapy & 8.73 & 0.784 \\
\hline & Control & 8.83 & 0.746 \\
\hline Mid-test pain & Total & 8.78 & 0.761 \\
\hline & Hypnotherapy & 4.78 & 0.897 \\
\hline Posttest pain & Control & 8.93 & 0.691 \\
\hline & Total & 6.85 & 2.24 \\
\hline Follow up Test Pain & Hypnotherapy & 2.66 & 0.802 \\
\hline & Control & 8.73 & 0.739 \\
\hline & Total & 5.70 & 3.153 \\
\hline & Control & 8.76 & 0.727 \\
\hline & Total & 5.65 & 3.251 \\
\hline
\end{tabular}

The results of this analysis regarding $F$ value, then the significance level of $F$ value showed that the model in betweensubjects and within subjects is significant (Table 3, table 4).

Table 3

Multivariate tests on the within-subject and between-subject effects (pain intensity)

\begin{tabular}{|lllllllll|}
\hline Source & $\begin{array}{l}\text { Sum of } \\
\text { squares }\end{array}$ & df & $\begin{array}{l}\text { Mean } \\
\text { Square }\end{array}$ & F & Sig. & $\begin{array}{l}\text { Partial Eta } \\
\text { Squared }\end{array}$ & $\begin{array}{l}\text { Noncent } \\
\text { Parameter }\end{array}$ & $\begin{array}{l}\text { Observed } \\
\text { Power }\end{array}$ \\
\hline Intercept & 10921.504 & 1 & 10921.504 & 19465.728 & 0 & 0.997 & 19465.728 & 1 \\
\hline Group & 1092.204 & 1 & 1092.204 & 1834.382 & 0 & 0.969 & 1834.382 & 1 \\
\hline Error & 32.542 & 58 & 0.561 & & & & & \\
\hline
\end{tabular}


Table 4

Multivariate tests on the within-subject and between-subject effects (DASS)

\begin{tabular}{|llllllllll|}
\hline Source & & $\begin{array}{l}\text { Sum of } \\
\text { squares }\end{array}$ & df & $\begin{array}{l}\text { Mean } \\
\text { Square }\end{array}$ & F & Sig. & $\begin{array}{l}\text { Partial } \\
\text { Eta } \\
\text { Squared }\end{array}$ & $\begin{array}{l}\text { Noncent } \\
\text { Paramete }\end{array}$ & $\begin{array}{l}\text { Observed } \\
\text { Power }\end{array}$ \\
\hline Intercept & Depression & 140070.017 & 1 & 140070.017 & 16949.256 & 0 & 0.997 & 16949.256 & 1 \\
& Anxiety & 141523.267 & 1 & 141523.267 & 32497.589 & 0 & 0998 & 32497.589 & 1 \\
\hline & Stress & 137808.338 & 1 & 137808.338 & 29380.643 & 0 & 0.998 & 29280.643 & 1 \\
\hline Group & Depression & 140106.667 & 1 & 140106.667 & 1706.986 & 0 & 0.967 & 1706.986 & 1 \\
\hline & Anxiety & 14508.150 & 1 & 14508.150 & 3331.466 & 0 & 0.983 & 3331.466 & 1 \\
\hline & Stress & 15120.938 & 1 & 15120.938 & 3212.801 & 0 & 0.982 & 3212.801 & 1 \\
\hline & Depression & 479.317 & 58 & 8.264 & & & & & \\
\hline
\end{tabular}

There are significant differences between the two groups. The efficacy of the procedure of hypnotherapy in the experimental group was more than that in the control group.

\section{Discussion}

In the present study, it was found that the group which underwent hypnotherapy showed a higher decrease in pain intensity than the control group. The data also showed that only the patients who underwent hypnotherapy, experienced a significant decrease in psychological distress. On the other word, the present study indicates that hypnotherapy affected both variables meaningfully, while control group didn't show any decrease in pain intensity and psychological distress among females with PMDD during 4 phases of assessments.

In consistent with this result, a systematic review reported that hypnotherapy decreased the use of sedative drugs and psychiatric drugs among patients with pain by decreasing their pain intensity $(18,19)$, however in the meantime it has been reported that restructuring attitude toward menstruation via increasing cognitive flexibility and improving patience have essential role in perception of pain and pain behaviors(20). In another study, it was found that physiotherapy cannot influence patients' viewpoints on pain, locus of control, and psychological indications, but affects pain intensity meaningfully $(9,12)$.

The other study also showed that hypnotherapy decreased psychological distress, and this decrease predicted the reduction in pain intensity in the near future among females with $\operatorname{PMDD}(20,21)$. In this manner some scientific investigations defy the effectiveness of hypnotherapy on psychological distress, especially stress subscale $(9,13,21)$. investigation showed that suggestibility and attitude to menstruation can significantly predict degree of pain intensity and psychological distress among female before and during menstruation, therefore hypnotherapy, cognitive hypnotherapy and psychoeducation focused on self-hypnosis can be applicable in treatment plans of females with $\operatorname{PMDD}(22,23)$. Regardless of therapeutic effects in which present study have investigated that, hypnotherapy and hypnotherapy focused psychoeducation can be used as an important effective program in prevention programs among females with PMDD(24).

It has been reported that one of the most important advantages of hypnotherapy focused psychotherapies is longitudinal therapeutic effects $(25,26)$. In consistent with literature, present study showed that hypnotherapy is capable to affect 
patients decreased psychological distress and pain intensity during long time after follow up assessment phase $(27,28)$. However, more definitive studies are needed for it to be a first-line intervention but there is sufficient evidence to suggest hypnotherapy as a viable treatment modality for persistent pain(24). Moreover In consistent with our results, meta- analysis gives strong support for the beneficial effect of hypnotherapy on anxiety and depression in women with premenstrual syndrome(7). It has been shown that Psychiatric conditions are common and can be effective on gynecologic conditions and their treatment outcomes, so it's essential to therapeutic approaches for psychiatric comorbidities in the gynecologic patient and psychotropics for nonpsychiatric conditions be discussed(3).

research has demonstrated that ego-strengthening suggestions and scripts can enhance treatment effects pain reduction plans among females with $\operatorname{PMDD}(29)$. The result of an investigation indicated that ego-strengthening-based hypnotherapy can be effective in reducing negative self-talk and negative attitudes, moreover it can improve cognitive flexibility(30). Moreover, short-term intensive comprehensive group psychotherapy with individuals with PMDD results in obtaining the desired changes in the cognitive functioning about restructuring attitudes manifested through the increase in ego strength(31). Early versions of ego-strengthening suggestions tended to be verbal and authoritarian in nature while later, developments added imagery and emphasized projective scripts with the purpose of accessing internal resources(13). Currently, in most hypnosis case studies and research articles, some form of ego-strengthening is an important aspect of the treatment plan(1, 32).

Findings confirmed large practical reductions of anxiety and depression among females with PMDD by group hypnotherapy focused on cognitive flexibility and its results were maintained at follow-up phase(32). In this manner it has been reported that high cognitive flexibility increases the female's capacity to cope with distress(33). Maternal cognitive perceptions can influence menstrual, premenstrual, labor and delivery related pain perception and should be considered when counseling patients with premenstrual difficulties. These findings support the mind-body correlation(34).

\section{Limitations:}

with regard to literature, it has been approved that incidence and intensity of symptoms especially pain and psychological distress is affected by personal attitudes toward menstruation, intergenerational educations and also cultural context, so one of the most important limitations of present study was investigation on sample of common national cultural of capital city of Iran.

\section{Suggestions:}

Future research can study effectiveness of hypnotherapy on PMDD symptoms with regard to cultural varieties. Moreover, its suggested that in future investigations, effectiveness of hypnotherapy on PMDD symptoms with regard to important others behavior and attitude to menstruation.

\section{Conclusion}

The present study findings revealed that hypnotherapy with suggestions focused on cognitive flexibility and ego strength affected pain intensity and psychological distress among females with PMDD significantly.

\section{Abbreviations}

PMDD

Premenstrual Dysphoric Disorder

PMS

Premenstrual Syndrome

DASS

Depression Anxiety and Stress Scales 
PSST

premenstrual symptoms screening tool

NRS

Numerical Rating Scale

\section{Declarations}

\section{Ethics approval and consent to participate:}

All of phases of study before conducting have been approved by ethical committee of Shahed University furthermore, all of participants have fulfilled conscious consent sheet at the first step of study.

\section{Consent for publication:}

Not applicable

\section{Availability of data and material:}

Information on where data supporting the results reported in the article can be found applicable.

\section{Competing interests:}

The authors declare that they have no competing interests.

\section{Funding:}

All sources of funding for the research have been accepted by corresponding author.

\section{Authors' contributions:}

Nader Abazari has performed practical tasks of study and writing the manuscript. Dr Leila Heydarinasab has designed the structure of study moreover accepted corresponding author. Dr Hamid Yaghubi supported theorical advices and in the end Dr Hojjatollah Farahani did data analysis by SPSS software.

\section{Acknowledgments:}

We appreciate all who contributed to this investigation especially females who underwent the research tasks. This study was confirmed and conducted for a Ph.D thesis in clinical psychology department of Shahed university of Tehran. Therefore, we acknowledge the assistance of the staff of Shahed university of Tehran

\section{References}

1. Beddig T, Reinhard I, Ebner-Priemer U, Kuehner C. Reciprocal effects between cognitive and affective states in women with Premenstrual Dysphoric Disorder: An Ecological Momentary Assessment study. Behaviour Research and Therapy. 2020:103613.

2. di Scalea TL, Pearlstein T. Premenstrual dysphoric disorder. Psychiatric Clinics. 2017;40(2):201-16.

3. Hofmeister S, Bodden S. Premenstrual syndrome and premenstrual dysphoric disorder. Am Family Phys. 2016;94(3):236-40.

4. Sepede G, Brunetti M, Di Giannantonio M. Comorbid premenstrual dysphoric disorder in women with bipolar disorder: management challenges. Neuropsychiatric Disease Treatment. 2020;16:415. 
5. Weise C, Kaiser G, Janda C, Kues JN, Andersson G, Strahler J, et al. Internet-based cognitive-behavioural intervention for women with premenstrual dysphoric disorder: a randomized controlled trial. Psychother Psychosom. 2019;88(1):16-29.

6. Wakatsuki Y, Inoue T, Hashimoto N, Fujimura Y, Masuya J, Ichiki M, et al. Influence of childhood maltreatment, adulthood stressful life events, and affective temperaments on premenstrual mental symptoms of nonclinical adult volunteers. Neuropsychiatr Dis Treat. 2020;16:1.

7. Cope GF. Premenstrual dysphoric disorder: A new psychological entity. Independent Nurse. 2019;2019(5):28-31.

8. Yen J-Y, Lin P-C, Huang M-F, Chou W-P, Long C-Y, Ko C-H. Association between Generalized Anxiety Disorder and Premenstrual Dysphoric Disorder in a Diagnostic Interviewing Study. International Journal of Environmental Research Public Health. 2020;17(3):988.

9. Moss D, McGrady A, Davies TC, Wickramasekera I. Handbook of mind-body medicine for primary care. Sage; 2003.

10. Heydarpour S, Dehghan F. Comparing the effectiveness of relaxation by hypnotherapy and virtual reality technology in alleviation of the symptoms of premenstrual syndrome. Cogent Psychology. 2019;6(1):1686962.

11. Fisher CY. Cyclic perimenstrual pain and discomfort and the role of complementary and alternative medicine in its treatment 2019.

12. Alladin A. Evidence-based cognitive hypnotherapy for depression. Contemporary Hypnosis. 2009;26(4):245-62.

13. Girman A, Lee R, Kligler B. An integrative medicine approach to premenstrual syndrome. American journal of obstetrics gynecology. 2003;188(5):56-65.

14. Bijur PE, Latimer CT, Gallagher EJ. Validation of a verbally administered numerical rating scale of acute pain for use in the emergency department. Acad Emerg Med. 2003;10(4):390-2.

15. Pirbaglou M, Katz J, de Souza RJ, Stearns JC, Motamed M, Ritvo P. Probiotic supplementation can positively affect anxiety and depressive symptoms: a systematic review of randomized controlled trials. Nutrition research. 2016;36(9):889-98.

16. Steiner M, Macdougall M, Brown E. The premenstrual symptoms screening tool (PSST) for clinicians. Archives of Women's Mental Health. 2003;6(3):203-9.

17. Kekecs Z, Roberts RL, Na H, Yek MH, Slonena EE, Racelis E, et al. Test-Retest Reliability of the Stanford Hypnotic Susceptibility Scale, Form C and the Elkins Hypnotizability Scale. 2019.

18. Saghebi Saeedi K, Abolghasemi A, Akbary B. The Effectiveness of Positive Thinking and Self-compassion Training on Cognitive Flexibility and Cognitive Failure in Girls With Primary Dysmenorrhea. 56-45:(1)6;2020.

19. Sengar K. GYNAE-C-PSYCH-OLOGY: NEED OF TODAY. Indian Journal of Clinical Psychology. 2016;43(1):1-4.

20. Domoney C, Vashisht A, Studd J. Premenstrual syndrome and the use of alternative therapies. Ann N Y Acad Sci. 2003;997(1):330-40.

21. Sheinfeld H, Gal M, Bunzel ME, Vishne T. The etiology of some menstrual disorders: a gynecological and psychiatric issue. Health Care Women Int. 2007;28(9):817-27.

22. Fitzgerald K. Women's Health and the Workplace: The Impact of the Menstrual Cycle. Universidade Autonoma de Lisboa (Portugal); 2015.

23. Micozzi MS, Dog TL. Women's Health in Complementary and Integrative Medicine E-Book: A Clinical Guide. Elsevier Health Sciences; 2004.

24. Kansagara D, Freeman M, Ayers C, Kondo K, Noonan K, O’Neil M, et al. Guided Imagery, Biofeedback, and Hypnosis: A Map of the Evidence. 2019.

25. Brooker E. Cognitive hypnotherapy and EMDR: The longitudinal effects on trait anxiety and music performance in advanced pianists. Advances in Complementary \& Alternative medicine. 2019;5(4).

26. Ravalia M. Can Clinical hypnotherapy be used as an adjunct or an alternative to conscious sedation in dentistry? BDJ In Practice. 2019;32(6):23-4. 
27. Tukaev R. The Integrative Theory of Hypnosis in the Light of Clinical Hypnotherapy. Hypnotherapy: IntechOpen; 2020.

28. Garba MH, Mamman M. Hypnosis and Hypnotherapy: The Role of Traditional Versus Alternative Approach. Hypnotherapy: IntechOpen; 2020.

29. Phillips M. It Takes More Than Two to Tango: Building Secure Attachment Through Hypnotic and Ego-State Relationships. Am J Clin Hypn. 2020;62(1-2):95-117.

30. Brooker E. Cognitive Hypnotherapy. Hypnotherapy: IntechOpen; 2020.

31. Kiarsis V. Understanding Ericksonian Hypnotherapy: Selected Writings of Sidney Rosen. Routledge; 2019.

32. Chén OY, Cao H, Reinen JM, Qian T, Gou J, Phan H, et al. Resting-state brain information flow predicts cognitive flexibility in humans. Scientific reports. 2019;9(1):1-16.

33. Annisa DF, Afdal A, Daharnis D, Adlya SI. Hypnotherapy as an alternative approach in reducing anxiety in the elderly. Konselor. 2019;8(1):32-7.

34. Spiegel EB, Baker EL, Daitch C, Diamond MJ, Phillips M. Hypnosis and the therapeutic relationship: Relational factors of hypnosis in psychotherapy. Am J Clin Hypn. 2020;62(1-2):118-37.

\section{Figures}




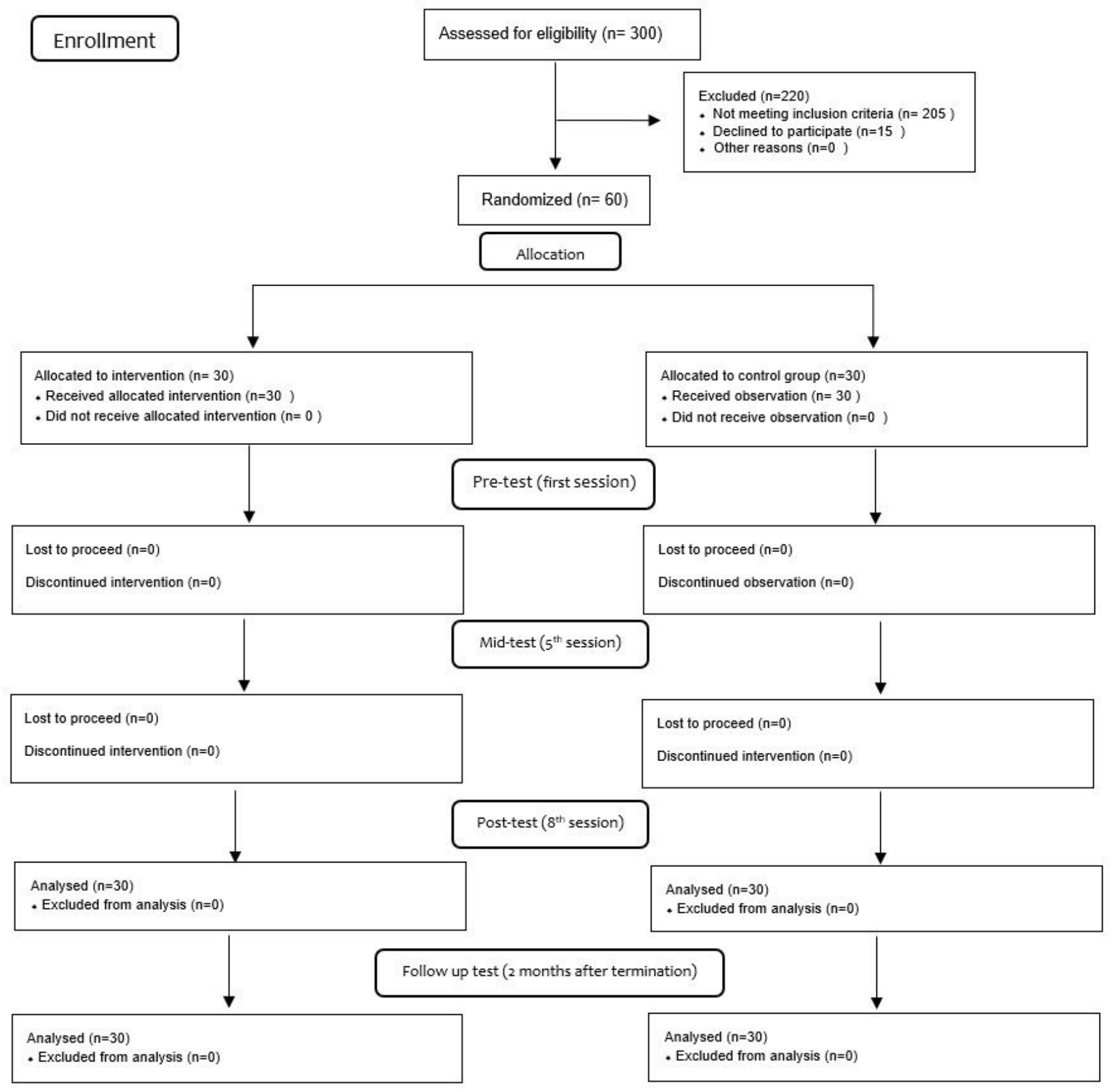

Figure 1

CONSORT Flow Diagram 